\title{
Investigating patient expectations and treatment outcome in a chronic low back pain population
}

This article was published in the following Dove Press journal:

Journal of Pain Research

27 January 2012

Number of times this article has been viewed

\author{
Kristen B Sanderson' \\ Daniela Roditi' \\ Steven Z George ${ }^{2}$ \\ James W Atchison ${ }^{3}$ \\ Evangelia Banou ${ }^{4}$ \\ Michael E Robinson' \\ 'Department of Clinical Health \\ Psychology, ${ }^{2}$ Department of Physical \\ Therapy, ${ }^{3}$ Department of Orthopedics \\ and Rehabilitation, University of \\ Florida, Gainesville, FL, USA; ${ }^{4}$ Chronic \\ Pain Rehabilitation Program, James A \\ Haley VA Hospital, Tampa, FL, USA
}

Correspondence: Michael E Robinson University of Florida, I0I South Newell Drive, Room 315I, PO Box 100165 , Gainesville, FL 32610-9165, USA

$\mathrm{Tel}+\mathrm{I} 3252735220$

Fax + I 352-273 6I56

Email merobin@ufl.edu
Objective: This study aimed to measure the outcomes that patients consider clinically meaningful across four treatment domains - (1) pain, (2) fatigue, (3) emotional distress, and (4) level of interference - and determine if patients met their own success criteria. Additionally, the role of expectations in treatment outcome was examined. This study also aimed to determine how change in levels of pain, fatigue, disability, and level of interference varied according to the type of treatment delivered to participants.

Patients: Forty-seven chronic low back pain patients were recruited from university-affiliated pain clinics.

Design: The study design was longitudinal, consisting of two randomly assigned treatment conditions. The first treatment condition used opioid medication only and the second used both opioid medication and brief cognitive behavioral therapy. Pre- and post-treatment assessments were conducted, which occurred approximately 3 months after the initiation of treatment.

Outcome measures: A patient-centered outcomes questionnaire was completed by participants at both pre- and post-treatment assessment.

Results: Results suggest that patients did not meet their own success criteria in treatment across any of the four domains. There was a significant main effect of time for level of pain indicating that both treatment groups had a decrease in their level of pain at post-treatment, $F(1,45)=11.98$, $P<0.001$. There was a significant main effect of time for level of interference domain indicating that both groups experienced a reduction in the level of pain-related interference with daily activities, $F(1,45)=5.46, P<0.05$. There were no significant effects of time for emotional distress or fatigue or any significant group by time interactions. Contrary to our hypothesis, no significant correlations were found between pretreatment expectations and usual level ratings at post-treatment across the four domains.

Conclusion: Patients sought larger reductions in pain, fatigue, level of distress, and level of interference than they attained at post-treatment. Enhancing opioid treatment with brief cognitive behavioral therapy did not yield additional improvements for the four domains assessed in patients with chronic low back pain.

Keywords: patient centered, chronic pain, expectations, treatment outcome, cognitive behavioral therapy

\section{Introduction}

The National Center for Health Statistics reported that one in four adult Americans stated that they experienced a period of pain lasting more than 24 hours within the previous month; one in ten people reported the pain being present for a minimum duration of 1 year. $^{1}$ In addition, approximately $25 \%$ of the US population reported experiencing chronic pain, ${ }^{1}$ with $18 \%$ of the population experiencing chronic low back 
pain at any given time. ${ }^{2}$ Due to its widespread prevalence, pain constitutes an economic burden to society as well as the individual, as is clearly shown by the annual direct and indirect expenditures that were recently reported from the Institute of Medicine, which reported that pain costs the nation between US\$560 and US\$635 billion each year in both medical treatment and lost productivity. ${ }^{3}$ The financial impact of low back pain alone ranges from US $\$ 45$ billion to US\$54 billion per year. ${ }^{4}$

Patients' pain experiences are often examined through self-report measures and behavioral observations. However, health care professionals also need to evaluate the degree to which patients want their pain and related domains to improve. A patient-centered outcomes questionnaire $(\mathrm{PCOQ})^{5}$ was developed to measure the patient-centered expectations and criteria for success regarding treatment for chronic pain.

A study by Robinson and colleagues found that, based on an eleven-point numerical rating scale where $0=$ "not at all important" and $10=$ "most important," patients deemed a mean reduction in their pain of 3.4 points $(56 \%)$ a successful treatment outcome. ${ }^{5}$ In addition, patients considered the following to be meaningful levels of success in treatment: a mean reduction of 3.4 points $(57 \%)$ in their level of fatigue, 3.6 points $(65 \%)$ in their level of overall distress, and 4.3 points $(68 \%)$ in the level of interference of pain in their lives. ${ }^{5}$ Patients' success criteria were shown to require a larger reduction in pain, distress, and fatigue than originally hypothesized, though patients did not expect to reach their own success criteria for the level of interference domain. ${ }^{5}$ Similarly, research has shown that, to consider treatment for pain successful, patients' criteria for treatment outcome are relatively high compared with health care professionals' criteria. .,7 $^{6,}$

Standard clinical assessments typically consist of measurements with a subjective cut-off score, or normative reference, also known as a minimal clinically important difference. The minimal clinically important difference has been used in pain studies in order to determine if patients change throughout treatment; it was found that a $30 \%$ change represented a clinically significant change in pain. ${ }^{8}$ However, reaching statistical significance (which may represent artificially inflated values based on a large sample size) does not unequivocally signify that patients have experienced a perceived successful degree of change. ${ }^{5,9}$ Several variables may influence findings, including sample size and degree of variability of improvement across patients, that is, greater improvement in some patients but deterioration in others. ${ }^{5,10}$
The minimal clinically important difference does not incorporate patients' criteria of treatment outcomes, which can identify group differences in patients, based on which domains they wish to target in treatment. It is evident that patients have different criteria by which they measure the success of their treatment. Therefore, health care professionals need to consult with patients to determine how their patients measure success in various domains, to determine how they can most effectively help their patient achieve this success.

A patient-centered approach focuses on patients' needs, views, and expectations, and incorporates them into treatment decisions. ${ }^{11}$ The Patient-Generated Index ${ }^{12}$ is one measure that incorporates the patient's perspective by examining the effect of a medical condition on quality of life; it also assesses the areas for improvement that are meaningful to the patient, and how realistic these expectations are in the areas of life patients want to change. In patient-centered models, patients have a more active and empowering role in structuring their treatment since they may have a different definition of success than their providers. Without patient involvement, success in treating pain is limited and patient suffering increases. ${ }^{13}$ With increased suffering, cost will likely rise as well.

This study aimed to first examine if patients who were treated at a multidisciplinary treatment center met their own success criteria in treatment at the 3-month follow-up assessment. Based on previous literature ${ }^{5}$ it was hypothesized that patients would not fully meet their own success criteria in treatment, as it is believed that they typically require larger reductions across domains in treatment than they will actually attain. Secondly, in addition to investigating patients' actual improvement against their success criteria, the researchers assessed the effect of brief cognitive behavioral therapy (CBT) and opioid treatments on expectations for treatment outcome from the patient perspective. It was anticipated that brief individualized CBT would have a greater influence on non-pain outcome expectations, whereas opioid treatment alone would primarily influence pain outcomes. Thirdly, we hypothesized that those who received brief CBT and opioid treatment would report greater actual success, as measured by the PCOQ, across the four domains, when compared with the opioid-only group, which would likely have a similar reduction in pain but lower levels of improvement in the other three domains (negative affect, fatigue, and interference with daily functioning). The relative greater success was hypothesized to be due to the cognitive restructuring aspects of brief CBT and the focus on behavioral pain management techniques, as compared to the opioid only group. 
Based on previous research, CBT has been successfully used as treatment for chronic low back pain populations. ${ }^{14}$ Finally, the fourth aim was to examine the extent to which patients' baseline expectations for treatment outcome predicted actual treatment outcome. Based on previous literature, ${ }^{15,16}$ it was hypothesized that there would be a positive correlation between expectation scores and outcomes.

\section{Methods}

\section{Participants}

Patients in the present study included 47 adults (20 female and 27 male) with chronic low back pain. Patients were recruited from the University of Florida Chronic Analgesic Management Program.

\section{Design}

This study had a two-group, pre-post design. The post-treatment assessments occurred 3 months after the initial assessment.

Inclusion criteria dictated that patients be between the ages of 18 and 70 and have low back pain as their primary pain site. Additionally, pain must have been present for a minimum of 3 months. Exclusionary criteria included a history of drug abuse or dependency within the past 5 years, presence of any medical condition where opioids were contraindicated, or psychosis.

\section{Procedure}

The University of Florida Institutional Review Board approved the procedures and protocols of the study. Informed consent was obtained from all participants. Patients were recruited and randomized to one of two conditions. The first condition consisted of brief individualized CBT and opioid medication, while the second condition consisted of opioid medication only. Both conditions were monitored weekly for 10 weeks for side effects.

All patients were on opioids, though the opioid medication varied according to individual prescriptions. Treatment condition assignment was conducted independent of medication usage. In terms of both short- and long-acting medication usage, no significant differences were found between the CBT group and the opioid-only group at baseline $\left(X^{2}[n=47\right.$, degrees of freedom $(\mathrm{df})=1]=1.52, P>0.05)$, nor at the 3 -month follow-up $\left(\mathrm{X}^{2}[\mathrm{n}=47, \mathrm{df}=1]=0.195, P>0.05\right)$. Patients randomized to the CBT and opioids group participated in brief CBT provided in an individualized format; thus, the length of therapy varied across patients. CBT sessions were each 1 hour in length and performed by therapists with specific training in the use of CBT with chronic pain. Each session consisted of a combination of skill and homework review, as well as new skill acquisition. Individualized CBT was utilized in order to tailor treatment to the patient and target domains that were clinically relevant to the individual. Skills learned in this brief intervention were taught according to individual needs; these included new ways to conceptualize pain (gate control theory); psychoeducation; pleasant activity scheduling; and examining the relationship between thoughts, feelings, and behaviors that may have been maintaining or exacerbating the patient's pain. Patients were also taught adaptive coping skills, such as activity pacing, and a variety of relaxation techniques.

\section{Measures}

\section{Demographic questionnaire}

Patients completed a demographic questionnaire inquiring about their age, sex, race/ethnicity, and number of years of formal education attained.

\section{PCOQ}

The PCOQ consists of four domains - (1) pain, (2) fatigue, (3) emotional distress, and (4) interference with daily activities - rated on a $0-100$ scale. Each of the four domains is measured on five levels: (1) usual levels, (2) desired levels, (3) treatment outcomes for success, (4) expectations of treatment, and (5) importance of improvements. Reliability for the PCOQ ranges from 0.84 to $0.90(P<0.001)$ for usual levels of pain, fatigue, distress, and interference. Recently published data regarding PCOQ psychometric properties demonstrated good concurrent validity. ${ }^{6}$

\section{Statistical methods}

All statistical analyses were conducted using SPSS ${ }^{\circledR}$ PASW 18 (IBM Corporation, Armonk, NY). For the first aim, addressing whether patients met their own success criteria, success criteria scores were calculated by finding the difference between patients' usual levels across domains and corresponding success criteria. In addition, actual change scores from pre- to post-treatment were calculated to see if patients matched their own success criteria, and then descriptive statistics were run. For the second and third aims of the study, repeated measures analyses of variance (ANOVA), under the general linear model, were performed to determine if there were significant differences found in each of the four domains from pre- to post-treatment, as well as between the two treatment groups. For the final aim, residualized change scores were used to determine the relationship between 
baseline expectations for change and actual outcome across the four domains.

\section{Results}

The final sample consisted of 47 adult patients; descriptive statistics are presented in Table 1. To address the first aim of the study, we examined success criteria as defined by patients, and then determined if patients met their own criteria. Patients indicated on a scale of $0-100$ that they considered treatment success with regards to their level of pain to be a mean reduction of 50.91 points in pain; however, on average, patients only reached a reduction of 11.93 points. This finding indicates that patients did not meet their own criterion. In addition, patients reported that their success criterion for overall distress would be a mean reduction of 34.62 points, though they instead experienced a mean 0.43 point increase in their level of emotional distress. In addition, patients reported that they sought a mean reduction of 40.62 points in the level of fatigue they were experiencing, though they had an actual decrease of 3.99 points in the fatigue domain. Lastly, patients indicated that success in their overall level of interference would be a mean reduction of 49.34 points; they experienced an actual mean reduction of 10.04 points. Results of dependent $t$-tests confirmed that these differences were statistically reliable $(P<0.001)$, indicating that patients did not meet their success criteria for any domain. These results are presented in Table 2.

Table I Participant demographics $(n=47)$

\begin{tabular}{llll}
\hline & Mean & SD & $\%$ \\
\hline Age & 48.23 & 9.89 & \\
Formal education (years) & 11.17 & 4.07 & \\
Duration of pain (months) & 138.41 & 107.55 & \\
Sex & & & \\
$\quad$ Female & 20 & & 42.6 \\
$\quad$ Male & 27 & & 57.4 \\
Race & & & \\
$\quad$ Caucasian & 38 & & 80.9 \\
$\quad$ African-American & 7 & & 14.9 \\
$\quad$ Hispanic/Latino & 2 & & 4.2 \\
Baseline "usual" levels & & & \\
$\quad$ Pain & 77.19 & 18.19 & \\
$\quad$ Fatigue & 61.53 & 23.94 & \\
$\quad$ Emotional distress & 48.23 & 31.72 & \\
$\quad$ Level of Interference & 67.45 & 28.78 & \\
Post-treatment "usual” levels & & & \\
$\quad$ Pain & 65.26 & 22.88 & \\
Fatigue & 57.64 & 25.87 & \\
Emotional distress & 48.28 & 28.36 & \\
Level of Interference & 57.4 & 30.18 & \\
\hline
\end{tabular}

Abbreviation: SD, standard deviation.
Table 2 Comparison of patients' treatment success criteria with actual improvements achieved

\begin{tabular}{lllll}
\hline $\begin{array}{l}\text { PCOQ } \\
\text { domain }\end{array}$ & $\begin{array}{l}\text { Patients' } \\
\text { success criteria } \\
\text { (mean) }\end{array}$ & $\begin{array}{l}\text { Reduction } \\
\text { attained } \\
\text { (mean) }\end{array}$ & $\begin{array}{l}\text { Cohen's } \\
\text { d }\end{array}$ \\
\hline Pain & 50.91 & 11.93 & $10.89^{*}$ & 3.21 \\
Emotional & 34.62 & -0.43 & $8.26^{*}$ & 2.44 \\
distress & & & & \\
Fatigue & 40.62 & 3.89 & $10.25^{*}$ & 3.02 \\
Interference & 49.34 & 10.04 & $8.91^{*}$ & 2.63 \\
\hline
\end{tabular}

Notes: Scale $=0-100 ; * P<0.001$.

Abbreviation: $\mathrm{PCOQ}$, patient-centered outcomes questionnaire.

To address the second aim of the study, which examined low back pain patients' change in expectation from pre- to post-treatment, a series of 2 (group) $\times 2$ (time) repeated measures ANOVA analyses were performed to assess differences for each of the four patient-centered outcome domains. Results indicated that there were no significant changes in the four domains across time, irrespective of group assignment. There were no significant group-by-time interaction effects across the patient-centered outcome domains. For detailed results see Table 3 .

For the third aim of the study, the change in usual level of each domain over time (from pre- to post-treatment) across the two treatment groups was examined using a repeated measures 2 (group) $\times 2$ (time) ANOVA. For the pain domain, results indicated that there was a significant effect of time, indicating that both groups reported a decrease in pain from pre- to post-treatment $(F[1,45]=11.98, P<0.001)$. For the interference domain, there was a significant main effect of time $(F[1$, $45]=5.46, P<0.05)$. These results indicate that both groups reported a significant decrease in their interference of daily living activities. There were no significant effects of time for emotional distress or fatigue. There were no significant group-by-time interactions for any of the analyses. For a complete report of results across PCOQ domains, see Table 4.

To determine a relationship between expectations and actual change (aim 4), residualized change scores were first calculated by regressing pretreatment scores on posttreatment scores for participants' usual levels across the four domains. Subsequently, correlations between patients' pretreatment expectations of treatment-induced change and their residualized change scores on usual levels of pain, fatigue, emotional distress, and level of interference were conducted. Results indicated that pretreatment expectations for change were not significantly correlated with the corresponding change of usual level of pain, fatigue, distress, 
Table 3 Patients' change in expectation from pre- to post-treatment

\begin{tabular}{|c|c|c|c|c|c|c|c|}
\hline \multirow[t]{2}{*}{ PCOQ domain } & \multicolumn{2}{|l|}{ CBT + opioids } & \multicolumn{2}{|l|}{ Opioids } & \multirow[t]{2}{*}{$\boldsymbol{F}$} & \multirow[t]{2}{*}{$\eta^{2}$} & \multirow[t]{2}{*}{$P$} \\
\hline & $\begin{array}{l}\text { Pretreatment } \\
\text { Mean (SD) }\end{array}$ & $\begin{array}{l}\text { Post-treatment } \\
\text { Mean (SD) }\end{array}$ & $\begin{array}{l}\text { Pretreatment } \\
\text { Mean (SD) }\end{array}$ & $\begin{array}{l}\text { Post-treatment } \\
\text { Mean (SD) }\end{array}$ & & & \\
\hline Pain & 28.46 (18.59) & 29.04 (20.39) & $28.57(25.50)$ & $37.38(28.00)$ & & & \\
\hline Time & & & & & 1.38 & 0.03 & $>0.05$ \\
\hline Group & & & & & 0.60 & 0.01 & $>0.05$ \\
\hline Time $\times$ group & & & & & 1.06 & 0.02 & $>0.05$ \\
\hline Fatigue & $23.85(18.18)$ & $20.19(17.41)$ & $23.10(25.71)$ & $26.19(24.23)$ & & & \\
\hline Time & & & & & 0.01 & 0.00 & $>0.05$ \\
\hline Group & & & & & 0.26 & 0.01 & $>0.05$ \\
\hline Time $\times$ group & & & & & 0.94 & 0.02 & $>0.05$ \\
\hline Emotional distress & $20.58(19.30)$ & $18.46(17.93)$ & $17.14(27.18)$ & $22.86(25.00)$ & & & \\
\hline Time & & & & & 0.26 & 0.01 & $>0.05$ \\
\hline Group & & & & & 0.01 & 0.00 & $>0.05$ \\
\hline Time $\times$ group & & & & & 1.23 & 0.03 & $>0.05$ \\
\hline Interference & $21.73(21.58)$ & I8.85 (I8.46) & $28.10(31.36)$ & $25.00(27.70)$ & & & \\
\hline Time & & & & & 0.48 & 0.01 & $>0.05$ \\
\hline Group & & & & & 1.14 & 0.03 & $>0.05$ \\
\hline Time $\times$ group & & & & & 0.00 & 0.00 & $>0.05$ \\
\hline
\end{tabular}

Abbreviations: CBT, cognitive behavioral therapy; PCOQ, patient-centered outcomes questionnaire; SD, standard deviation.

or level of interference. See Table 5 for a report of correlations across domains.

\section{Discussion}

The first aim of the present study examined whether patients met their own treatment success criteria by the end of treatment. Results indicated that, overall, patients did not meet their own success criteria, a finding consistent with previous literature. ${ }^{5}$ Consistent with our hypothesis, the amount of change necessary for patients to consider their treatment successful was of much greater magnitude than the change actually achieved with treatment. Patients' treatment-related improvements did not meet their success criteria for any of the four domains examined. In addition, this shows that opioids are not effective in successfully treating chronic low back pain. The failure of treatment to meet

Table 4 Patients' change in usual levels from pre- to post-treatment

\begin{tabular}{|c|c|c|c|c|c|c|c|}
\hline \multirow[t]{2}{*}{ PCOQ domain } & \multicolumn{2}{|l|}{ CBT + opioids } & \multicolumn{2}{|l|}{ Opioids } & \multirow[t]{2}{*}{$\boldsymbol{F}$} & \multirow[t]{2}{*}{$\eta^{2}$} & \multirow[t]{2}{*}{$P$} \\
\hline & $\begin{array}{l}\text { Pretreatment } \\
\text { Mean (SD) }\end{array}$ & $\begin{array}{l}\text { Post-treatment } \\
\text { Mean (SD) }\end{array}$ & $\begin{array}{l}\text { Pretreatment } \\
\text { Mean (SD) }\end{array}$ & $\begin{array}{l}\text { Post-treatment } \\
\text { Mean (SD) }\end{array}$ & & & \\
\hline Pain & $74.73(18.47)$ & $68.08(19.19)$ & $80.24(17.8 I)$ & $61.76(26.84)$ & & & \\
\hline Time & & & & & $11.98^{* *}$ & 0.21 & $<0.001$ \\
\hline Group & & & & & 0.01 & 0.00 & $>0.05$ \\
\hline Time $\times$ group & & & & & 2.65 & 0.06 & $>0.05$ \\
\hline Fatigue & $60.96(22.32)$ & $59.04(24.78)$ & $62.24(26.35)$ & $55.90(27.68)$ & & & \\
\hline Time & & & & & 0.98 & 0.02 & $>0.05$ \\
\hline Group & & & & & 0.02 & 0.00 & $>0.05$ \\
\hline Time $\times$ group & & & & & 0.28 & 0.01 & $>0.05$ \\
\hline Emotional distress & $53.58(31.42)$ & $53.27(25.96)$ & $41.62(31.58)$ & $42.10(30.57)$ & & & \\
\hline Time & & & & & 0.00 & 0.00 & $>0.05$ \\
\hline Group & & & & & 2.31 & 0.05 & $>0.05$ \\
\hline Time $\times$ group & & & & & 0.01 & 0.00 & $>0.05$ \\
\hline Interference & $65.00(29.16)$ & 57.31 (28.78) & 70.48 (28.72) & $57.52(32.54)$ & & & \\
\hline Time & & & & & $5.46^{*}$ & 0.11 & $<0.05$ \\
\hline Group & & & & & 0.14 & 0.00 & $>0.05$ \\
\hline Time $\times$ group & & & & & 0.35 & 0.01 & $>0.05$ \\
\hline
\end{tabular}

Notes: ${ }^{*} P<0.05 ;{ }^{* *} P<0.001$.

Abbreviations: $\mathrm{CBT}$, cognitive behavioral therapy; PCOQ, patient-centered outcomes questionnaire; SD, standard deviation. 
Table 5 Pretreatment expectation and pre- to post-treatment change in usual level ratings

\begin{tabular}{lll}
\hline & $\boldsymbol{r}$ & $\boldsymbol{P}$ \\
\hline Change in pain & 0.08 & $>0.05$ \\
Change in emotional distress & 0.03 & $>0.05$ \\
Change in fatigue & 0.02 & $>0.05$ \\
Change in level of interference & -0.02 & $>0.05$ \\
\hline
\end{tabular}

Note: Degrees of freedom $=45$.

patients' success criteria may explain why patients often try numerous therapies and medication approaches; perhaps they are dissatisfied with current treatment approaches that do not beget improvements corresponding to their own success criteria for treatment.

The present study further explored patient-centered assessment and outcomes across four functional domains. The PCOQ was originally designed to measure various domains of functioning in a chronic pain population. This study administered the measure to a chronic low back pain sample in order to examine specific expectations this sample may have had, and to determine how expectations may have changed over time. This study sought to examine if chronic low back pain participants' expectations for change within the four domains differed from pre- to post-treatment based on group assignment.

This hypothesis was based on the premise that the constructs of CBT would effect changes in expectations through cognitive restructuring; therefore, it was hypothesized that the opioid-only group would not experience systematic changes in expectations with regard to level of interference, fatigue, or distress as compared to the brief CBT and opioids group. Contrary to our hypothesis, as well as to previous research on the role of expectations ${ }^{17}$ there were no significant differences between groups or across time for all other domains. The lack of findings on a role of expectations could be attributed to a power issue, due to a small sample size. As seen in Table 3, effect sizes are quite small. Since patients did not meet their own criteria for success, it is possible that expectations did not significantly change because patients still had expectation for a reduction in pain and related domains even after treatment had ended; they might have hoped to still experience change. Based on what is implied in the instructions, the PCOQ may not assess patients' perceptions of the magnitude of improvement achieved at treatment termination but may reflect how much they will continue to improve following treatment.

This study also aimed to determine how change in usual levels of pain, fatigue, disability, and level of interference varied according to the type of treatment delivered to participants. No differences in usual levels of pain, fatigue, disability, or level of interference were found between the two treatment groups. A main effect of time was found in the pain domain, indicating that both treatment groups improved significantly from pre- to post-treatment, though the expected benefit from brief CBT beyond that of the opioid-only treatment was not supported.

Lastly, this study aimed to determine how expectations influence actual change. However, our results did not confirm our original hypothesis that there would be a correlation between baseline expectations and outcome of usual levels of pain, fatigue, distress, and level of interference. Given the small changes in all domains relative to patient criteria for success, this may have minimized the expectation and actual change relationship. This sample was particularly chronic and previous experience with treatments may have limited their expectations for success.

Few patient-centered approaches for the evaluation of expectations and treatment outcome in a chronic pain population have been examined. The PCOQ offers a novel approach to examining chronic low back pain patients' changes in expectation and provides important information to contribute to the pain literature.

Although the study had significant strengths, it is crucial to address some potential limitations. One potential limitation of the study may be the delivery and duration of the CBT. The current study employed standard clinical care but did not follow a manualized treatment protocol, which may have reduced the effectiveness of the CBT. Future studies may consider providing manualized CBT of longer duration. Future research should investigate the usefulness of CBT, as compared with opioids, by adding another treatment group where patients receive CBT only so that results can be more conclusive. Reliability and validity of the PCOQ can be further examined in future studies to better ensure that chronic low back pain patients' domains of functioning are being measured in a meaningful and accurate way. Finally, this study was limited in that it had a sample size of only 47 participants.

\section{Conclusion}

Results replicated previous findings indicating that patient criteria for success are not met by opioid and CBT-enhanced opioid treatment. As far as the authors are aware, the present study is the most recent to show that current treatments fall considerably short of patients' criteria for success. Future directions for development of chronic pain treatment 
necessitate the establishment of more effective analgesic strategies, or more targeted interventions aimed at more realistic expectations for current treatments. Patients' expectations at pretreatment did not predict treatment outcome, probably because the magnitude of change was quite low, and the group may have had low expectations based on the chronicity of their pain. It is important that the expectations of individuals with chronic low back pain, and how these relate to their treatment outcome, are investigated in the future. The information collected will help inform clinical treatment (eg, expectations may need to be targeted as part of treatment or addressed more specifically in treatment) with the goal of attaining future improvements that can facilitate a greater degree of pain relief and pain-related sequelae for chronic low back pain patients.

\section{Acknowledgment}

This research was supported by grant R01NR010324 from the National Institute for Nursing Research to Michael Robinson.

\section{Disclosure}

The authors declare no conflicts of interest in relation to this paper.

\section{References}

1. National Centre for Health Statistics. Health, United States, 2006, with Chartbook on Trends in the Health of Americans. Hyattsville, MD: National Center for Health Statistics; 2006.

2. Turk DC. Clinical effectiveness and cost-effectiveness of treatments for patients with chronic pain. Clin J Pain. 2002;18(6):355-365.

3. National Academy of Sciences Institute of Medicine. Relieving Pain in America: A Blueprint for Transforming Prevention, Care, Education, and Research. Washington, DC: National Academy of Sciences; 2011.
4. National Academy of Sciences Institute of Medicine. Musculoskeletal Disorders and the Workplace: Low Back Pain and Upper Extremeties. Washington, DC: National Academies Press; 2001.

5. Robinson ME, Brown JL, George SZ, et al. Multidimensional success criteria and expectations for treatment of chronic pain: the patient perspective. Pain Med. 2005;6(5):336-345.

6. Brown JL, Edwards PS, Atchison JW, Lafayette-Lucey A, Wittmer VT, Robinson ME. Defining patient-centered, multidimensional success criteria for treatment of chronic spine pain. Pain Med. 2008;9(7):851-862.

7. Thorne FM, Morley S. Prospective judgments of acceptable outcomes for pain, interference and activity: patient-determined outcome criteria. Pain. 2009;144(3):262-269.

8. Farrar JT, Young JP Jr, LaMoreaux L, Werth JL, Poole RM. Clinical importance of changes in chronic pain intensity measured on an 11-point numerical pain rating scale. Pain. 2001;94(2):149-158.

9. Farrar JT. What is clinically meaningful: outcome measures in pain clinical trials. Clin J Pain. 2000;16(2 Suppl):S106-S112.

10. Farrar JT, Portenoy RK, Berlin JA, Kinman JL, Strom BL. Defining the clinically important difference in pain outcome measures. Pain. 2000;88(3):287-294.

11. Laine C, Davidoff F. Patient-centered medicine. A professional evolution. JAMA. 1996;275(2):152-156.

12. Ruta DA, Garratt AM, Leng M, Russell IT, MacDonald LM. A new approach to the measurement of quality of life. The Patient-Generated Index. Med Care. 1994;32(11):1109-1126.

13. Teh CF, Karp JF, Kleinman A, Reynolds Iii CF, Weiner DK, Cleary PD. Older people's experiences of patient-centered treatment for chronic pain: a qualitative study. Pain Med. 2009;10(3):521-530.

14. Reid MC, Otis J, Barry LC, Kerns RD. Cognitive-behavioral therapy for chronic low back pain in older persons: a preliminary study. Pain Med. 2003;4(3):223-230.

15. Mahomed NN, Liang MH, Cook EF, et al. The importance of patient expectations in predicting functional outcomes after total joint arthroplasty. J Rheumatol. 2002;29(6):1273-1279.

16. Kalauokalani D, Cherkin DC, Sherman KJ, Koepsell TD, Deyo RA. Lessons from a trial of acupuncture and massage for low back pain: patient expectations and treatment effects. Spine (Phila Pa 1976). 2001;26(13):1418-1424.

17. Constantino MJ, Manber R, Ong J, Kuo TF, Huang JS, Arnow BA. Patient expectations and therapeutic alliance as predictors of outcome in group cognitive-behavioral therapy for insomnia. Behav Sleep Med. 2007;5(3):210-228. 


\section{Appendix}

\section{Patient-centered outcomes questionnaire}

Many people experience pain, fatigue (ie, feeling tired), emotional distress (eg, worries, feeling sad), and interference with daily activities (eg, not being able to work or do household chores) as a result of their medical condition. We would like to understand how you have been impacted in each of these areas. We would also like to learn more about what you want your treatment to do for you.

First, we would like to know your usual levels of pain, fatigue, emotional distress, and interference.

On a scale of $\boldsymbol{0}$ (none) to $\mathbf{1 0}$ (worst imaginable), please indicate your usual level (during the past week) of ...

- pain

- fatigue (or tiredness)

- emotional distress

- interference with daily activities

Now, we would like to learn about your desired levels of pain, fatigue, emotional distress, and interference. In other words, we would like to understand what your ideal treatment outcome would be.

On a scale of $\mathbf{0}$ (none) to 10 (worst imaginable), please indicate your desired level of ...

- pain

- fatigue (or tiredness)

- emotional distress

- interference with daily activities

Journal of Pain Research

\section{Publish your work in this journal}

The Journal of Pain Research is an international, peer-reviewed, open access, online journal that welcomes laboratory and clinical findings in the fields of pain research and the prevention and management of pain. Original research, reviews, symposium reports, hypothesis formation and commentaries are all considered for publication.
Patients understandably want their treatment to result in desired or ideal outcomes like you indicated above. Unfortunately, available treatments do not always produce desired outcomes. Therefore, it is important for us to understand what treatment outcomes you would consider successful.

On a scale of $\boldsymbol{0}$ (none) to $\mathbf{1 0}$ (worst imaginable), please indicate the level each of these areas would have to be at for you to consider treatment successful.

- pain

- fatigue (or tiredness)

- emotional distress

- interference with daily activities

Now, we would like to know what you expect your treatment to do for you.

On a scale of 0 (none) to 10 (worst imaginable), please indicate the levels you expect following treatment.

- pain

- fatigue (or tiredness)

- emotional distress

- interference with daily activities

Finally, we would like to understand how important it is for you to see improvement in your pain, fatigue, emotional distress, and interference following treatment.

On a scale of 0 (not at all important) to 10 (most important), please indicate how important it is for you to see improvement in your...

- pain

- fatigue (or tiredness)

- emotional distress

- interference with daily activities

\section{Dovepress}

The manuscript management system is completely online and includes a very quick and fair peer-review system, which is all easy to use. Visit http://www.dovepress.com/testimonials.php to read real quotes from published authors. 